\title{
Commission 5. (BIBLIOGRAPHIE)
}

I. La Commission demande que la subvention attribuée à la "Bibliographie mensuelle" soit portée de $£$ IOO à $€ I 50$.

2. C'est avec plaisir que la Commission apprend que le Comité National Belge a trouvé les moyens de permettre au Dr Pelseneer d'entreprendre et de poursuivre le travail du regretté Dr Stroobant pour combler la lacune entre la Bibliographie de Houzeau-Lancaster et le Jahresbericht. La Commission sera heureuse d'aider de toute manière le Dr Pelseneer dans son entreprise.

3. La Commission recommande l'emploi de la classification décimale pour la littérature astronomique.

4. La Commission a désigné le professeur Vyssotsky, du Leander McCormick Observatory, pour faire les démarches nécessaires en vue d'une traduction de la partie astronomique des anciennes chroniques russes.

5. La Commission demande à l'Observatoire de Lund de dresser un catalogue cartographique des astronomes et des observatoires.

6. C'est avec plaisir que la Commission apprend que le Dr $\mathrm{H}$. von Klüber, de l'Observatoire de Potsdam, a l'intention d'étudier, conjointement avec des égyptologues experts, les documents de l'astronomie égyptienne antique.

7. La Commission a décidé de préparer une courte liste de sujets relatifs à l'histoire de l'astronomie. Quand cette liste sera prête, des dispositions seront prises pour sa diffusion parmi les astronomes et les hommes de lettres.

8. La Commission demande que, en accord avec la Société Royale de Londres, des dispositions soient prises pour rassembler et publier les Lettres Scientifiques de Isaac Newton, et cela, si possible, avant 1942 .

\section{Commission 6. (TELtGgammes)}

La Commission recommande l'attribution au Bureau des Télégrammes astronomiques, pour les trois prochaines années, d'une subvention annuelle de I200 francs or.

\section{Commission 9. (INSTRUMENTS)}

La Commission émet le vœu suivant:

Les auteurs d'instruments destinés à des recherches astronomiques, doués de propriétés optiques nouvelles, sont priés de donner toujours l'indication complète des données de construction, sous une forme qui permette un examen critique et éventuellement la reproduction: indices et dispersion des matières employées, courbures des surfaces, épaisseurs et distance des lentilles et, si possible, description des aberrations résiduelles et des propriétés photométriques.

\section{Commission IO. (TAChES SOLAIRES ET NOMBRES CARACTÉRISTIOUES)}

I. Que le titre Bulletin for Character Figures of Solar Phenomena soit remplacé par Quarterly Bulletin on Solar Activity.

2. Que l'on supprime, à l'avenir, dans le dit Bulletin, les données relatives à la zone centrale, mais que l'on continue à publier, pendant le temps où elles peuvent paraître de quelque utilité pour les recherches statistiques, celles qui se rapportent au disque entier. 